\title{
3D car shape reconstruction from a contour sketch using GAN and lazy learning
}

\author{
Naoki Nozawa ${ }^{1} \cdot$ Hubert P. H. Shum ${ }^{2,3}(\mathbb{D}) \cdot$ Qi Feng $^{1} \cdot$ Edmond S. L. $\mathrm{Ho}^{2}$ (I) $\cdot$ Shigeo Morishima ${ }^{4}$
}

Accepted: 5 September 2020 / Published online: 16 April 2021

(c) The Author(s) 2021

\begin{abstract}
3D car models are heavily used in computer games, visual effects, and even automotive designs. As a result, producing such models with minimal labour costs is increasingly more important. To tackle the challenge, we propose a novel system to reconstruct a 3D car using a single sketch image. The system learns from a synthetic database of 3D car models and their corresponding 2D contour sketches and segmentation masks, allowing effective training with minimal data collection cost. The core of the system is a machine learning pipeline that combines the use of a generative adversarial network (GAN) and lazy learning. GAN, being a deep learning method, is capable of modelling complicated data distributions, enabling the effective modelling of a large variety of cars. Its major weakness is that as a global method, modelling the fine details in the local region is challenging. Lazy learning works well to preserve local features by generating a local subspace with relevant data samples. We demonstrate that the combined use of GAN and lazy learning produces is able to produce high-quality results, in which different types of cars with complicated local features can be generated effectively with a single sketch. Our method outperforms existing ones using other machine learning structures such as the variational autoencoder.
\end{abstract}

Keywords Generative adversarial network $\cdot$ Lazy learning $\cdot 3$ D reconstruction $\cdot$ Sketch-based interface $\cdot$ Car $\cdot$ Contour sketch

Supplementary Information The online version contains supplementary material available at https://doi.org/10.1007/s00371020-02024-y.

Hubert P. H. Shum

hubert.shum@durham.ac.uk

Naoki Nozawa

s112800563@akane.waseda.jp

Qi Feng

fengqi@ruri.waseda.jp

Edmond S. L. Ho

e.ho@northumbria.ac.uk

Shigeo Morishima

shigeo@waseda.jp

1 Department of Pure and Applied Physics, Waseda University, Tokyo, Japan

2 Department of Computer and Information Sciences, Northumbria University, Newcastle upon Tyne, UK

3 Department of Computer Science, Durham University, Durham, UK

4 Waseda Research Institute for Science and Engineering, Waseda University, Tokyo, Japan

\section{Introduction}

3D car models are heavily used across multiple fields such as entertainment [14], visual effects [42] and automotive designs [50]. The process to generate models that resemble similar features from the real-world ones is usually timeconsuming and labour-intensive. Automatic approaches that reconstruct 3D models from a single image input can be served as effective solutions.

Despite significant research [10,50] in related areas, highquality reconstruction of complicated 3D car models remains challenging due to several reasons. First, in animation, game, and automotive manufacturing industries, the design process usually involves concept arts of the car, which are typically represented as sketches on predefined viewpoints. Therefore, previous methods with photo inputs [25] are not practical in designing new shapes or modifying existing designs. Second, for learning-based methods, different types of cars such as SUVs and trucks consist of significantly diverse features, making the process of learning complicated data distributions difficult for a single neural network [36]. Third, cars modelling with fine details is a distinct problem as cars have common features such as where to place the wheels, but also 


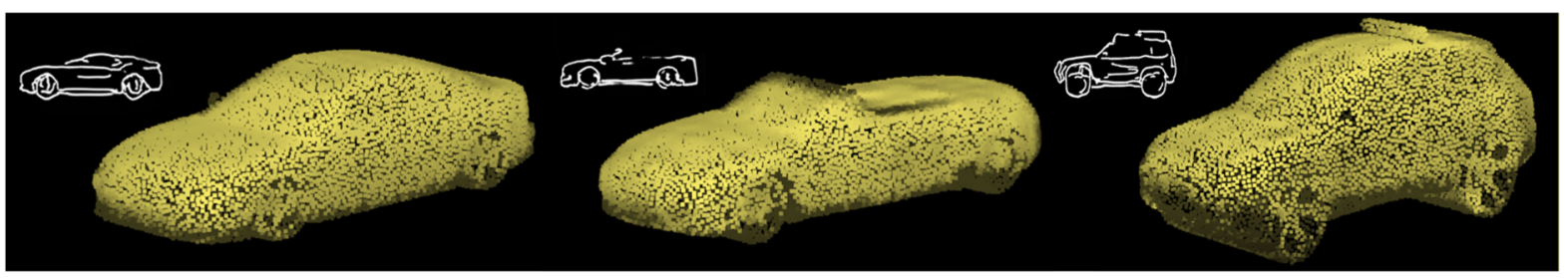

Fig. 1 Examples of 3D car shapes generated by our system with side-view sketches. Top-left white lines are input contour sketches, while blue point clouds are corresponding outputs

distinctive parts such as the shape of rear wings and roofs [10]. Past research [50] shows that it is challenging to learn a diverse car subspace that represents both common and distinctive car features well.

In this paper, we propose a novel system to reconstruct a 3D car using a single sketch image, enabling an effective car shape creation process. To provide a good user interface for creating car shape, we use contour sketches [31] that contain car boundaries and salient inner edges as the input. Such drawing greatly helps the car designing process as it allows users to directly use their understanding of scene geometry. We propose a data generating system that produces synthetic quadruplets of contour sketches, depth, segmentation masks and shape annotations from 3D car models obtained from ShapeNet [4] for facilitating an effective training process and a feature-preserving car mesh augmentation pipeline to maximize the data variation.

To tackle the challenge of modelling 3D car shapes, we propose a novel two-stage machine learning framework. In the first stage, we propose a GAN-based network that learns from contour sketches and 3D shapes to ensure a wide variety of car shapes. As the GAN [15] has shown success in modelling 3D shapes [43] with its strong ability to model complicated data distributions, we adapt GAN to generate global shapes of 3D car models. Since it is computationally inefficient to directly learn 3D shape representation from mesh, we propose to learn an intermediate representation of multiple depth images instead and reconstruct the 3D car mesh as a post-processing step. As global deep learningbased methods are limited in representing local details such as rear wings [16,50], in the second stage, we introduce a lazy learning method to learn a local subspace from the relevant samples in the database. Compared to traditional approaches, lazy learning postpones the generalization of the database to run-time [3], which reduces the scale of learning by only considering the most relevant data. This facilitates the representation of local features that may be insignificant on a global scale. We further apply principal component analysis (PCA) to improve the search space of point clouds, from which we search for the $\mathrm{k}$ nearest neighbours. We finally perform a low-cost optimization process on the subspace to generate a 3D car shape with fine details.
Experimental results show consistent outputs of 3D car models generated from contour sketches with diverse shapes and topologies (Fig. 1). In addition to well-resembled global shapes of contour sketches, fine details and local features such as rear bumpers are also effectively preserved in the generated models (Fig. 8). When compared to existing methods, ours outperforms existing ones using other machine learning structures such as the variational autoencoder (VAE) [37].

The major contributions of this paper are summarized as follows:

- We propose a system to synthesize training data to construct a large database of 56,224 samples with contour sketches, depth, masks and 3D models. With realistic sketch-like features, the contour sketch facilitates realworld designing and editing applications.

- We propose a generative adversarial network to learn the correlation between a $2 \mathrm{D}$ contour sketch and the corresponding multi-view depth images that generate a 3D shape. Our GAN-based method outperforms existing learning-based approaches such as VAE with more diverse car topologies and shapes.

- We propose a lazy learning algorithm to learn a local subspace to reconstruct the fine detail features of the car. Such a subspace bases only on the relevant car shapes in the database and therefore effectively retains detailed features in the samples [4].

The preliminary results of this work have been presented in [37]. In this work, we have made the following technical improvements. First, we represent a new database using contour sketches for a better sketch-like drawing style, while [37] uses a naive Laplacian filter to generate artificial samples. Second, we propose GAN that can more robustly learn the $3 \mathrm{D}$ representation of different car types due to its strong capability to model complicated data distributions, as opposed to [37] that used VAE with limited capacity on varied car topologies. Third, we evaluate the proposed system with a set of new experiments. We further compare our work with [37] to demonstrate the improvements.

The rest of the paper is organized as follows: we review previous work in Sect. 2. We explain the construction of our 
car database with contour sketches in Sect. 3. We present our generative adversarial network for generating 3D car shapes from 2D sketches in Sect. 4. We present our lazy learning for constructing fine details for the car in Sect. 5. We show the results of our system in Sect. 6. We conclude the paper and discuss possible future directions in Sect. 7.

\section{Related work}

In this section, we first review previous sketch-based applications, followed by related work in the areas of sketch-style image rendering, data representations for 3D shapes, and finally machine learning for 3D shape reconstruction.

\subsection{Sketches for 3D reconstruction}

Sketches are powerful representations to capture users' design for computer graphics applications. In particular, we are interested in the problem in using sketches as a cue to reconstruct 3D shapes. Earlier work utilizes sketches with a predefined control interface to capture complicated 3D shape designs [23]. Such a method is further extended to improve the smoothness of the generated 3D shape [22], as well as building the internal structure of the shapes [39]. The control scheme was further enhanced to incorporate extra information that represents shape symmetry and angles [13]. A major problem for these methods is that the control scheme has to be manually designed, and users have to learn such schemes before using the system.

As an improvement, more general sketch-to-3D methods are proposed by using the input sketches to represent geometric features of the 3D shapes. For example, it is possible to use fit 3D primitive shapes into the input curves [47], to represent 3D inflating surfaces [26], or even to estimate the normal map of the surface of a shape [44]. That said, these methods aim at using artificial intelligence to infer the sketch information provided by the users. With advanced machine learning such as deep learning becoming more and more available, we prefer to learn such a kind of logics automatically.

To create sketch images effectively for machine learning approaches, sketch-style rendering techniques are useful. These techniques use 2D lines to represent 3D shapes. Different visual cues such as image boundaries and edges are usually inferred from a single input image with edge detectors such as [2]. However, these methods capture high-frequency signals without understanding the image. Boundary detectors, otherwise, understand the scene and yield semantic segmentation of different objects [34]. However, object boundaries that only contain outer edges poorly resemble realistic drawing features. Recently, contour sketches [31] are proposed to provide more sketch-like features while maintaining the ability to convey geometric information, salient edges and occlusion events. Contrary to professional computer-aided design software that requires professional training and has an engineering focus, sketch-based interfaces [38] are more designer-friendly. To accommodate users with diverse drawing skills and artistic styles, we adapt contour sketches as the input of our approach.

\subsection{Machine learning for 3D shape reconstruction}

3D shapes can be represented in different formats, and such representations affect the performance of machine learning approaches heavily. In general, there are three types of representation. The 3D point cloud has been used heavily for modelling 3D shapes due to its simplicity, enabling applications such as shape reconstruction [11] and shape classification [41]. The disadvantage of the representation is that it does not represent volumetric information. As a solution, voxels are used as they can model the volumetric occupancy of a complex 3D shape, allowing more accurate shape reconstruction [7]. However, although methods are proposed to relax the computation requirements using octrees [49], 3D operations are still computationally expensive. Depth images that represent different views of a 3D shape using 2D distance images can resolve this problem. As the surface information of a shape can be represented using multiple $2 \mathrm{D}$ views, $2 \mathrm{D}$ operations can be used to reconstruct 3D shapes [29], thereby significantly reduces the computational requirements. The combined use of depth images and normal images can further enhance the representation power and give details to the surfaces during a reconstruction process [24]. In this project, we utilize depth images as an intermediate output, such that we can relieve our system from using computationally expensive and difficult to optimize 3D operations. Multiple views of depth images are then combined to form a 3D shape using an analytical solution.

Traditional methods use multiple views to 3D reconstruct a scene. Reconstructing shapes from a single image is a challenging task but would benefit a wide range of real-world applications. With recent advances in deep learning, datadriven methods have gained increasing attention. Han et al. [17] propose a convolutional neural network (CNN)-based system to generate 3D faces from input sketches. Nishida et al. [36] adapt a CNN to generate building models by adding surface curve information as a style of sketching. In the preliminary work [37], We also utilize deep learning for constructing the sketch-based interface by adapting the Variational Autoencoder (VAE) [27] for correlating the 2D sketch and the output represented as depth and mask images. Although such a generative model has shown promising results in the translation of image style, its capability in modelling complicated data distribution is limited.

As a global model, GAN was introduced as a generative model to synthesize new instances from multiple predefined 
classes [15]. Other than computer graphic tasks such as image inpainting [40] and texture transfer [30], GAN has shown success in recovering the geometric structure from a single given image $[25,43]$. When reconstructing the $3 \mathrm{D}$ shapes from image inputs, the adversarial loss of GAN is capable of working as a fidelity regularizer and ensures that the generated samples share a close shape probability distribution with the training data. Considering the ability to learn complicated data distribution and the flexibility to support different inferences, we adapt GAN in our method to infer and generate global features of car models.

To further complement the expressiveness and represent fine details of the shapes that are specific to a small cluster of samples [50]. In the area of car reconstruction, different categories of cars have different specific details such as side mirrors and rear wings. Past research has shown that local models utilizing lazy learning can help to preserve fine details in different problems. Chai et al. [3] generate a human surface from a sparse input with a large motion database. Shum et al. [48] reconstruct noisy 3D human motion captured by Kinect using lazy learning. The main idea is to extract relevant data based on a run-time query and construct a local model during run-time. In this work, we adapt lazy learning to generate the fine details of a car based on the output generated by a deep learning network.

\section{Database creation}

In this section, we present a robust and efficient process to construct a 3D car mesh database with contour sketches that highly resemble human sketches. A synthesis-based approach reduces the cost to acquire expensive paired $2 \mathrm{D}$ and 3D training samples, while the generated database can be easily extended with a larger size and scale. We generate contour sketches with a conditional GAN and create a point cloud representation from the $3 \mathrm{D}$ meshes. With contour sketch annotations, it is made easier for users with drawing skills to make use of the approach. With novel feature-preserving data augmentation techniques, we create a large variety of logically correct car meshes.

The database contains two sets of representations: (1) 2D contour sketches, depth and mask images for shape reconstruction, and (2) registered 3D point clouds for details synthesis.

\subsection{Feature-preserving car mesh augmentation}

We follow the method in [37] to augment the 3D car meshes such that we can generate a larger database with more car variations. The key advantage of the method is that it can retain the local features of the car during the augmentation process. For example, while scaling a car, unlike previous
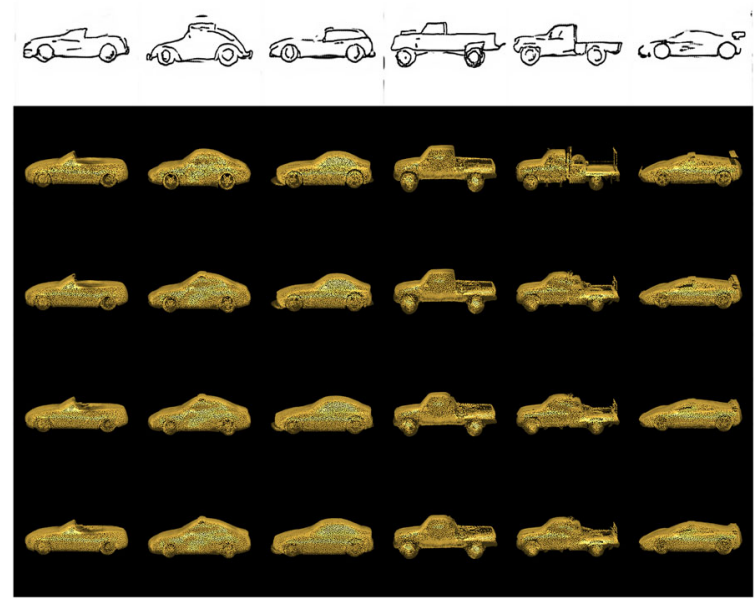

Fig. 2 Results generated with different $k$. From top to bottom: sketches, point clouds with $k=1, k=3, k=5$ and $k=7$

methods [43] that would distort the proportion of the car wheels, ours can maintain the circular wheel shape.

Here, we summarize the augmentation method. Readers are referred to [37] for more details.

Our car meshes came from ShapeNet [4], as it offers different models of car with different variations. That said, the database is not big enough to effectively train the deep learning system. Therefore, we augment the car meshes following [28], which allows us to change the shape of the car while retaining local features at the same time. The core of the method is to voxelize the mesh and to interpolate the vertices based on the augmented (i.e. scaled) voxel grid. In our system, the resolution of the grid is set as $5 \times 10 \times 15$. We then scale the grid with $\pm 20 \%, \pm 15 \%, \pm 10 \%$ and $\pm 5 \%$ for the height and length directions. With an input of 7,028 cars meshes, with our augmentation method, we can create 56,224 meshes. Examples are shown in Fig. 2.

\subsection{The 2D contour sketches depth and masks representation}

With the 3D meshes created, we produce the corresponding 2D contour sketches and depth representations for training our deep learning system on car shape reconstruction. On the one hand, traditional edge detector such as a Canny edge detector [2] only capture high-frequency signals of an input image, and such samples with excessive details greatly differ from human drawings. On the other hand, segmentation models are usually trained with only the outer boundaries with no salient inner edges, resulting in oversimplified predictions that poorly represent geometric information of the input [31]. To make our method practical when applied to real-world scenarios such as automotive designing processes, we synthesize contour sketches, which contain both the outer boundaries 
and salient inner edges to represent occlusion events happen in the original photorealistic counterpart, generated 3D meshes.

To achieve high-quality contour sketch inferences from 3D car meshes, we adapt conditional GAN [35]. Compared to a traditional GAN structure, we incorporate an additional L1 loss that compares the ground truth contour sketches with the predictions in addition to the adversarial loss of GAN. For generating contour sketches of cars with imperfect alignments, we freeze the weight that is trained with the contour sketches database [31] and predict sketches with rendered images from certain perspectives (front, top, side) with a copper material which empirically best resemble the samples from the previous database and then downsize the normalized cube faces to a $256 \times 256$ resolution for a more efficient generation process.

To create the depth and mask images, we set up a template cube that contains the normalized complete car shapes and then obtain depth and rendered images from each face of the cube. For efficient calculation, we utilize a pixel shader and store them as floating-point values. We ignore the bottom face of the car and do not produce the corresponding depth and mask. This is because the bottom of the car typically consists of complicated geometry involving mechanical gears, which is unrelated to our application.

Compared to existing databases that poorly resemble realistic sketches such as the Laplacian filter-based approach [37], our mesh augmentation framework creates high-quality sketch-like annotations and generates a new database that solves the limitation of sub-optimal performance when the trained model is applied to real-world scenarios.

\subsection{The registered 3D point cloud representation}

We follow [37] to generate a registered 3D point clouds format from the car meshes. The importance of the registration process is that it allows machine learning systems to have a uniform input vector for effective learning. In our system, this is particularly helpful when we use lazy learning introduce fine details into the car shape. We will give a summary of the method here. Readers are referred to [37] for the implementation details.

We fist generate a point cloud format using Poisson sampling [8]. As we are aiming for a registered point cloud format, we need to have the same number of points per car. We control the total number of points by iteratively changing the radius of the Poisson sampling process. Once the number is within an acceptable range, we randomly take away points such that the number of point reaches a predefined value, which is set as 10,000 in our system.

We then register the point clouds from different cars by considering this process as an Earth Mover problem $[19,46]$. This means that we will first select one point cloud randomly
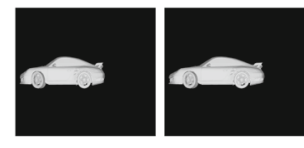

Z-axis - $20 \%$ Z-axis - $10 \%$

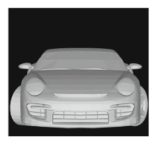

Y-axis $-20 \% \quad$ Y-axis $10 \%$

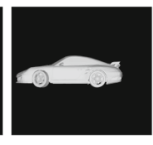

Original
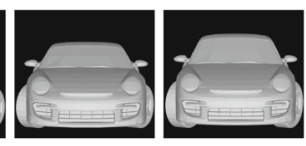

Original

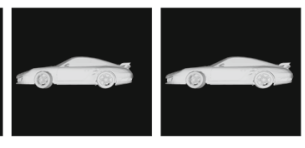

Z-axis $+10 \%$ Z-axis $+20 \%$

Y-axis $+10 \%$ Y-axis $+20 \%$

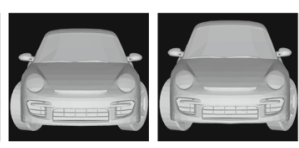

Fig. 3 Examples of 3D car meshes synthesized with our featurepreserving data augmentation method

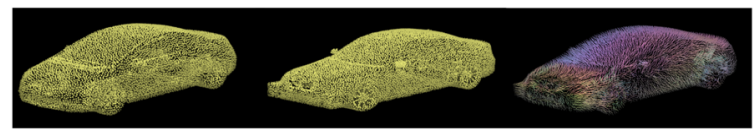

Fig. 4 From left to right: the template, a car shape, and the flow for mapping them

as the template, while treating all the rest as targets. For each point in the template point cloud, we will find the best mapping point in the target point cloud, such that the total distance between all template-target point pairs is minimized. Such one to one mappings for all points between the template and the target point clouds are called flow. Figure 3 visualizes the flow between the template and the target.

\section{Generative adversarial network for car shape reconstruction}

In this section, we present a deep neural network to reconstruct 3D car shapes from 2D contour sketches. With a GAN-based network, our method is capable of modelling complicated and distinctive shapes with an effective training process, and has the ability to generate high-quality shapes from a single contour sketch.

In the first stage of car shape reconstruction, we adapt Generative Adversarial Network (GAN) [15] for getting the depth images and reconstruct a rough 3D shape that resembles the 2D contour sketches, as such a generative model has shown promising results in image translation by altering the input with a different style. After the shapes of cars are generated, we introduce the details of the car in the process of the second stage.

\subsection{The design of the generative adversarial network}

We propose a GAN-based network that learns from contour sketches and 3D shapes to ensure a wide variety of car shapes. Compared to previous designs such as Variational Autoencoder (VAE) [27], GANs share superior performance 
$[6,24,51]$ in terms of the appearance of the output. More importantly, our novel network design takes input at the latent vector layer and generates multiple views from random noises and thus reduces the expensive training cost and allows a larger variety of car shapes. To further highlight the distinctive features among different car shapes during the reconstruction such as spoilers and rear wings, instead of directly outputting the $3 \mathrm{D}$ point cloud $[5,11]$ or the voxels [7,9] of a car, we propose to output a set of depth images from the side, top, front and rear views, and reconstruct the $3 \mathrm{D}$ vertices by combining them.

We adapt an encoder-decoder network structure as the generator for creating depth images [29]. We modified the design of the generator to add noise directly to the latent space, as shown in Fig. 4. The decoder needs to generate depth images in multiple predefined views. On the one hand, existing research typically prepares multiple decoders, with one decoder generating one output view [33]. However, such an approach increases computational cost and memory requirement significantly, considering that we need to generate five different views (i.e. front, rear, left, right, and top). On the other hand, traditional cGAN networks add noises to the input through concatenation, resulting in inefficient memory usage with increased input resolutions.

As a solution, our network shares the encoder among multiple views, and we novelly control the input at the latent vector layer to solve both limitations. This design is driven by the observation that there is shared information across different views. By sharing the same encoder, such information can be discovered. Apart from the massive reduction in memory usage and training time, such a set-up allows the different output views to be more coherence and produces higher quality results. We justify our choice in the network design by conducting an ablation test in Sect. 6.3.

\subsection{The loss function}

For the depth images, we implement two loss functions- the mean absolute error (MAE, L1 loss) on the generated depth images and the MAE on their Laplacian representations as in our pilot study [37]. In particular, minimizing the MAE loss on the generated depth images can preserve the overall shape and structure of the car. On the other hand, including the MAE loss on the Laplacian representation can better preserve the surface appearance. Readers are referred to [37] for more details and justifications.

An adversarial loss is added to the discriminator. By concatenating all views of depth images and input to the discriminator, the model learns to distinguish if the set of depths is real or not. Masks are one of the sub-task outcomes for depths, so we ignored such mask images when training the discriminator. By training a single discriminator for mul- tiple views, this design can save memory usage and learn the relationship between views simultaneously.

The final loss function is expressed as:

$$
\begin{aligned}
E= & \left.\left(\boldsymbol{D}^{\mathrm{ref}}-\boldsymbol{D}^{\mathrm{rec}}\right) \circ \boldsymbol{M}^{\mathrm{ref}}\right)_{L 1}+\left(\boldsymbol{M}^{\mathrm{ref}}-\boldsymbol{M}^{\mathrm{rec}}\right)_{\mathrm{BCE}} \\
& \left.+\left(\left(\Delta \boldsymbol{D}^{\mathrm{ref}}-\Delta \boldsymbol{D}^{\mathrm{rec}}\right) \circ \boldsymbol{M}^{\mathrm{ref}}\right)\right)_{L 1}+\text { ADVLoss }
\end{aligned}
$$

$$
\begin{aligned}
\mathrm{ADV} \text { Loss }= & \arg \min _{G} \max _{D}\left\{\log D\left(\boldsymbol{S}, \boldsymbol{D}^{\mathrm{ref}}\right)\right. \\
& +\log (1-D(\boldsymbol{S}, \boldsymbol{G}(\boldsymbol{S}, z))\}
\end{aligned}
$$

where $\boldsymbol{D}^{\text {ref }}$ and $\boldsymbol{M}^{\text {ref }}$ are depth and mask images of the ground truths, $\boldsymbol{D}^{\text {rec }}$ and $\boldsymbol{M}^{\text {rec }}$ are those of reconstructed images, $\boldsymbol{S}$ is input Sketch image, the subscripts $L 1$ and BCE (binary cross-entropy) represent the calculation metrics, $\Delta$ means Laplacian filtering and $\circ$ is the Hadamard product, ADVLoss is the adversarial loss function, $G$ is the generator, $D$ is the discriminator, and $z$ is the random noise vector.

\subsection{Surface reconstruction}

In this section, the process for reconstructing a rough point cloud from the generated depth and mask images generated by our proposed framework is presented.

As in our pilot study [37], the mask and depth images pairs from each view can be used for reconstructing the $3 \mathrm{D}$ point cloud of a part of the car. By aligning the parts reconstructed from different views, the overall shape of the car can be created (Fig. 5 (left)) as a single point cloud. The surface of the entire car (Fig. 5 (middle)) can then be reconstructed by applying Poisson surface reconstruction to the point cloud of the car. With the surface of the car, we can uniformly sample points from it and this step is equivalent to the point cloud standardization process as in the database creation (Sect. 3.3). By this, the register point cloud (Fig. 5 (right)) can be directly compared with the example cars in our database and similar cars will be used in the lazy learning stage (Sect.5) to add fine details to the car shape.

\subsection{Implementation details}

Our framework is implemented with Tensorflow. For optimization, we use Adam solver with a learning rate 1e-5. The decoder has a dropout ratio of 0.5 except for the last layer. Inspired by pix 2pix [24], We use Leaky ReLU as the activation function for the hidden layers in the encoder, and ReLU for that in the decoder. We use $\tanh$ as the activation function for output layers. More details regarding the network architecture can be referred to in Fig. 5. To achieve high efficiency, the resolution of the images that are inputted in GAN is $64 \times 64$. To ensure an accurate evaluation with unaltered 


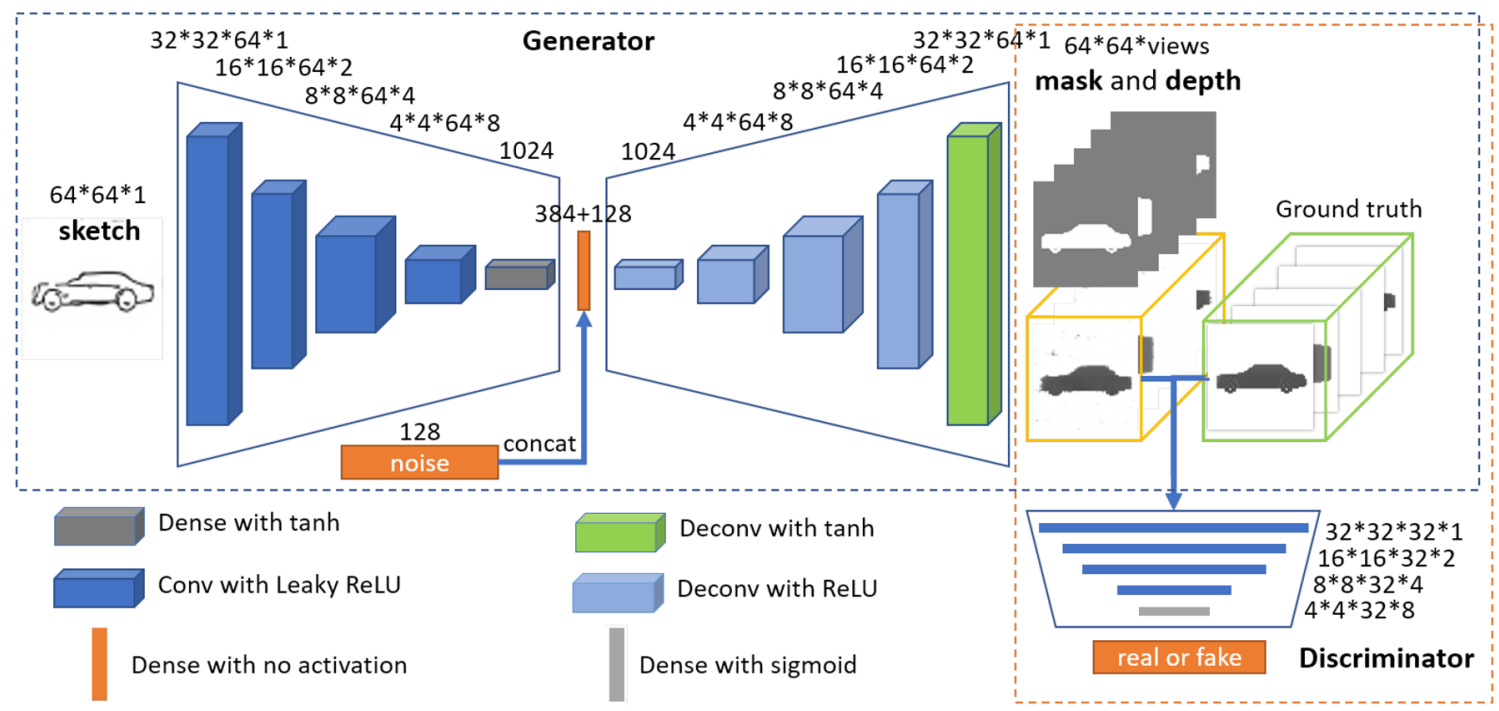

Fig. 5 The design of our generative adversarial network

data, we train the system using the data generated by data augmentation, and test the system with the original data from ShapeNet [4].

\section{Lazy learning for fine details}

We follow our preliminary work [37] in developing lazy learning algorithms to reconstruct local details. Here, we give a summary of the algorithms and highlight the important system designs. We refer the readers to [37] for the implementation details.

While the main bodies of cars share a lot of common geometric similarities, the fine details such as side mirrors can be different. Learning a universal model from all cars with fine details is therefore highly ineffective. Motivated by the success of lazy learning in mesh processing [3,20,45], we propose to adapt lazy learning to reconstruct the details.

Unlike traditional approaches that generalize data in the whole database as a preprocess, lazy learning postpones the generalization to run-time [3]. As a result, it can utilize run-time information to limit the scale of learning. In particular, given a run-time query, relevant data in the database can be extracted and a small-scale learning process can be performed. By only considering the most relevant data, the common features that may be insignificant on a global scale can be successfully represented. Besides, the similarity of relevant data allows lazy learning to use a much lower dimensional latent space comparing to traditional methods.

\subsection{Relevant data search}

Given a car shape generated in Sect.4, we search for the most relevant samples from the database and perform lazy learning. As the point cloud is registered (i.e. it aligns with a predefined template car shape), we can effectively calculate the distance using the sum of Euclidean distances from all points between two point clouds. To reduce the high dimensionality of the point clouds during searching, we propose to apply Principal Component Analysis (PCA) onto the position of the point clouds to generate a search space, instead of using the Cartesian space. Searching with the more important components of PCA allows a faster search with less focus of fine details. Following [37], we set the root mean square distances of the 40 PCA components to find $k=5$ nearest neighbours to achieve good results.

\subsection{Learning and optimization in local space}

With the $k$ nearest neighbours selected from the database, we can then learn a small subspace with PCA. Since these neighbours are similar to each other, the details of the shape can be well preserved with a smaller number of components. In such a subspace, we optimize a set of eigenvalues to construct a car shape that is as similar as possible to the one generated by deep learning. We then back-project the eigenvalues to formulate a car shape with details such as the headlight, which is served as our final output.

We utilize the 3D morphable model [1] to optimize the eigenvalues of the components with a nonlinear optimization process. Since the point clouds are registered, we use a simple point-to-point Euclidean distance to evaluate the distance between the optimizing shape and target shape in the Cartesian space. To obtain the Cartesian representation of the optimizing shape, we simply back-project the optimized eigenvalues to the Cartesian space. 


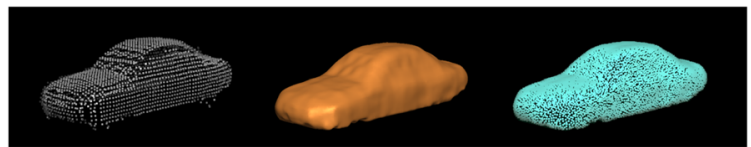

Fig. 6 From left to right: the reconstructed shape, the reconstructed surface, and the registered point cloud
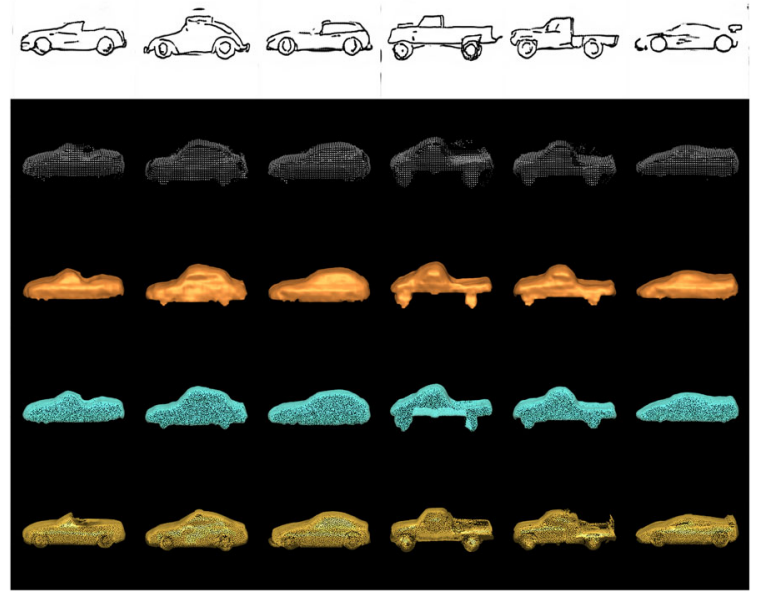

Fig. 7 Intermediate outputs. From top to bottom: sketches, meshes from generated depth images, reconstructed surfaces, sampled point clouds on surfaces, and point clouds with details

We propose a simple preprocess that constructs a more representative local PCA space with the $k$ nearest neighbours to further improve the optimization process. Based on the observation that there are still small variations in car shapes within the $k$ nearest neighbours, which distracts the system from the main objective of obtaining the detailed shape features, we pre-optimize these shapes individually using the same morphable model-based optimization process described above, such that they share a similar shape before we construct the local PCA space. This way, the significant components of the local PCA space can be more representative on the detailed shape features (Fig. 6).

\section{Experimental results}

We will first present the experimental results on reconstructing $3 \mathrm{D}$ car shape from input sketches. Next, we quantitatively analyse the training loss during the training process to show the convergence of the proposed framework. Finally, a comparison with the state-of-the-art method [37] will be presented to demonstrate the results obtained from different network architectures and justify our choice.

The training of the deep learning system is performed with an NVIDIA GeForce RTX 2080 Ti GPU that has 11GB VRAM. With the batch size of 32, the training finishes within a few days. The run-time system is performed on a lower- end computer with an NVIDIA GeForce 1050 Ti GPU that has 4GB VRAM. The reconstruction of a car takes approximately 15 seconds to finish, with 5 seconds on car shape reconstruction (i.e. deep learning) and 10 seconds on reconstruction detail features (i.e. lazy learning).

\subsection{Reconstructing 3D shape from contour sketches}

Since different users may have different drawing styles (e.g. more cartoon-like), real-world sketches are not objective to evaluate the performance of the proposed system. As a result, we utilize synthetic contour sketches for testing.

Examples of the output yielded by every major step of the proposed framework are illustrated in Fig. 7. Stating with the input sketch (top row), depth images are computed for reconstructing the 3D meshes (second row). Next, a smooth surface is reconstructed from the mesh (third row) and a point cloud (fourth row) is sampled from the surface for retrieving similar car shapes from the database for details refinement. Finally, the refined car model (bottom row) is created using the proposed lazy learning module. It can be seen that the meshes (Fig. 7, second row) reconstructed from the proposed GAN framework can already resemble the car shape specified in the abstract input sketch. The proposed lazy learning module further enhances the quality of the 3D models by adding details such as side mirrors and spoilers (Fig. 7, second row). This highlights the effectiveness of the proposed framework. Readers are referred to Fig. 1 and the video demo accompanied for more examples.

However, details like grilles or wheels are not encoded well for practical use of games or movies. The EMD registration process can cause such low-quality appearances because the EMD is based on the theory of optimal transport with global distribution, which can ignore small features. Besides, the converting process into point clouds can reduce mesh resolution that is closely related to details. We will consider landmarks on 3D mesh in the sampling and hierarchical registration process for encoding such small features. Furthermore, the input sketches can affect appearance because of sparse information comparing with photorealistic images. Feature extraction from sketches is still an open problem in the field of deep learning, so we will update our network structure. An interactive sketch-based system will improve appearance as well.

While the point clouds generated from the proposed framework is highly realistic in terms of the overall 3D shape, the system is less effective in reconstructing meshes with sharp edges. The underlying problem is related to the $3 \mathrm{D}$ point sampling process during the $3 \mathrm{D}$ mesh registration. The $3 \mathrm{D}$ point sampling tends to sample points around sharp edges instead of along the edges, which is a well-known problem in $3 \mathrm{D}$ point sampling [21]. As a result, the sharp edges may be lost when the 3D surface is reconstructed from the sampled 

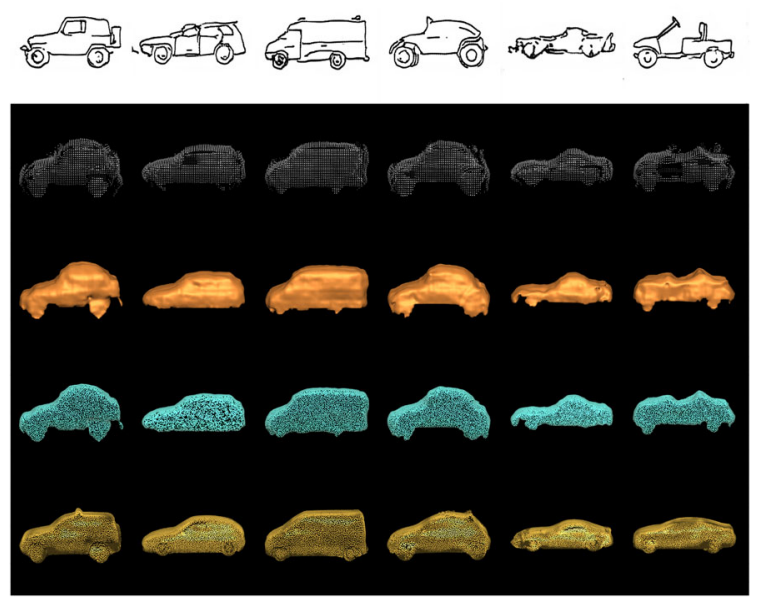

Fig. 8 Lower-quality results for sketches that have few similar samples in the database

points using triangulation. While this is an interesting topic to explore, this is out of the scope of this work. Exploring the feasibility of using more advanced sampling methods such as [12] or [18] can be an interesting future direction to further enhance the quality of the reconstructed 3D meshes.

We further conducted an experiment to have an in-depth analysis of the proposed lazy learning module. The proposed lazy learning module plays an important role in adding fine details such as the wheels and grills to the reconstructed 3D car shape. Here, we focus on the $k$-value that defines how many nearest neighbour will be selected for learning the local space (Sect.5). Different $k$-values are being used in this experiment, and the results are presented in Fig. 8. From the results, it can be seen that the points clouds tend to be more noise and contain more holes when $k=1$ and $k=3$ (Fig. 8, second and third rows). In addition, wrong details can be added to the final $3 \mathrm{D}$ point clouds as the lazy learning process is biased to a small number of samples when the $k$-value is small. For example, the shape of the bed of the truck on the fifth column (from left to right) in Fig. 8 is different from the input sketch when $k=1$ and $k=3$. On the other hand, the 3D point clouds generated using $k=7$ (Fig. 8, bottom row) do not have the aforementioned artefacts. However, car shapes tend to be over-smoothed. Finally, using $k=5$ (Fig. 8, fourth row) can generate a smooth surface while resembling the shape specified in the input sketch.

While our proposed framework can generate realistic car shapes from sketch images, some of the low-quality results are presented in Fig. 9 for further analysis. We found that low-quality results are usually associated with the car shapes that are uncommon in the data set. The underlying issue is related to the lack of similar car shapes for the refinement in the lazy learning step. For example, the 3D mesh (Fig.9, second row) generated by the proposed GAN framework has similar shapes as in the input sketch. However, the refined point clouds (Fig. 9, bottom row) changed the shape of the cars, especially the leftmost and rightmost columns in Fig. 9.

\subsection{Training loss}

Here, we present the results of a quantitative evaluation of the performance of the training process in the proposed framework. A wide range of training loss plots are illustrated in Fig. 6. The plots also contain the training losses obtained using 4 variants of the proposed decoder network as an ablation study and more detailed will be given in Sect. 6.3.

In particular, the depth loss, mask loss, Laplacian loss, and total loss are reduced stably as training progress. This highlights the proposed deep learning framework converges and can effectively improve the quality of the generated mesh in the training process. For the generator and discriminator losses (Fig. 6d, e), the oscillations are mainly caused by the competitive nature between the generator and discriminator, which is a typical pattern in GAN frameworks and the losses show a decreasing trend in general.

\subsection{Comparing with the state-of-the-art and ablation tests on different decoder network architectures}

In this section, we present the result of an ablation study to justify our network design and followed by comparing our results with those obtained using the state-of-the-art method [37]. As explained in Sect. 4.1, decoders are used for generating depth and masks in different views for reconstructing the 3D shape of the car from input sketches. While the images in different views have a different appearance, they are associated with the same underlying 3D shape. As a result, Nozawa et al. [37] proposed sharing a common layer among the decoders in the network design to preserve the underlying structure and improve the consistency among all synthesized views. In our proposed encoder-decoder network (Fig.4), each decoder consists of five layers. In the ablation test, we vary the number of shared layers in the decoder from 0 (i.e. not sharing any layer) to 3 . The different decoder architectures are illustrated in Fig. 10.

A wide range of 3D car shapes are reconstructed using different decoder network architectures, and the results are illustrated in Fig.11a. It can be seen that our proposed decoder architecture without sharing any layer (second row in Fig. 11) produces the best results in terms of reproducing the car shape with a smooth surface. On the other hand, sharing layers (third, fourth and fifth rows in Fig. 11) result in 3D point clouds with less distinguishable shape and noisy/rough surface, which can be caused the loss of balance between preserving the underlying structure among decoders and refining each view. On the other hand, Nozawa et al. [37] reported that results can be obtained using a decoder network with 1 


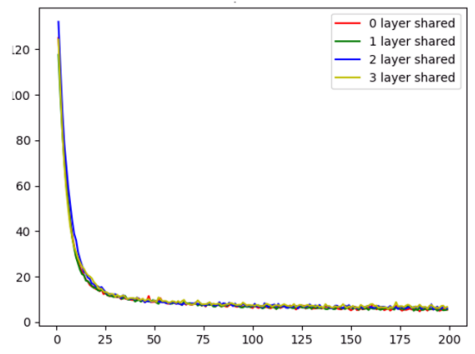

(a) Depth Loss

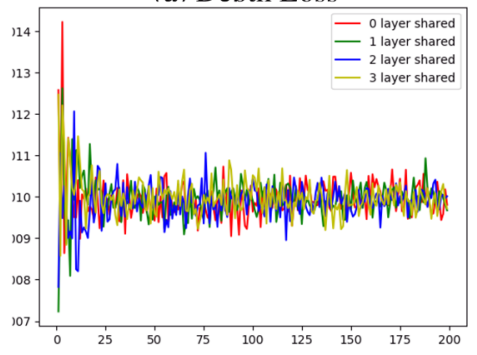

(d) Generator Loss

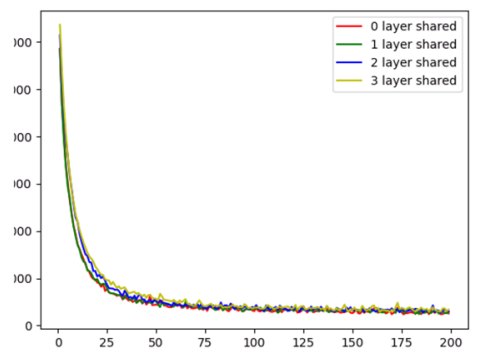

(b) Mask Loss

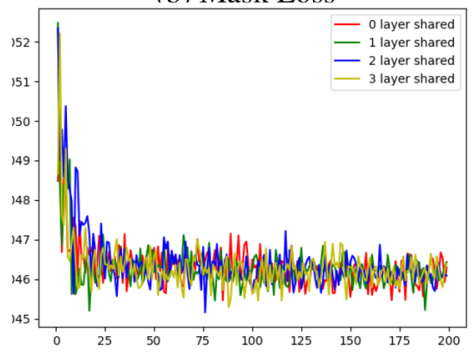

(e) Discriminator Loss

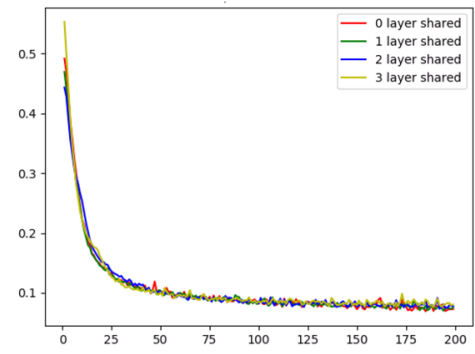

(c) Laplacian Loss

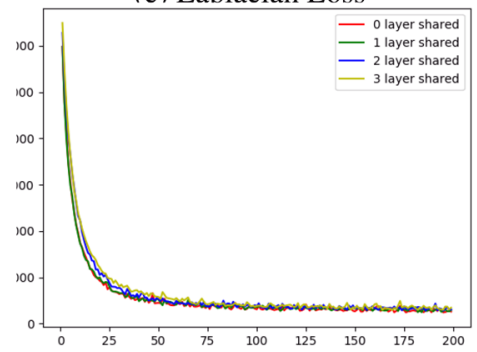

(f) Total Loss

Fig. 9 Losses across epoch during the training stage

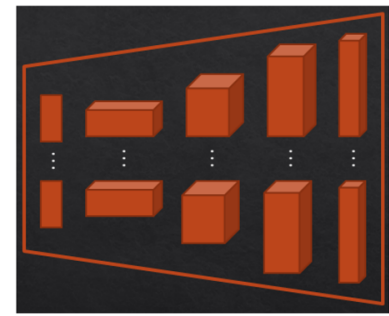

(a) No shared layer

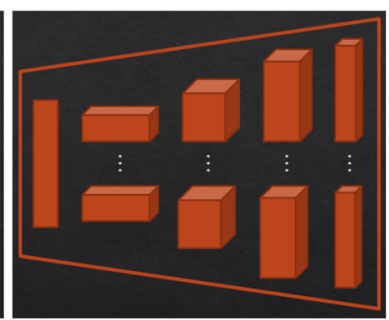

(b) A one-layer shared

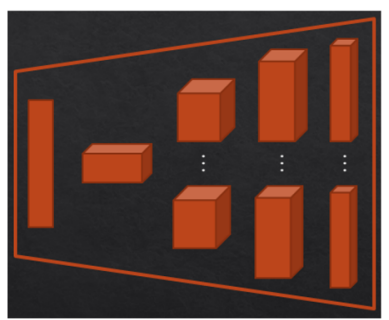

(c) Two layers shared

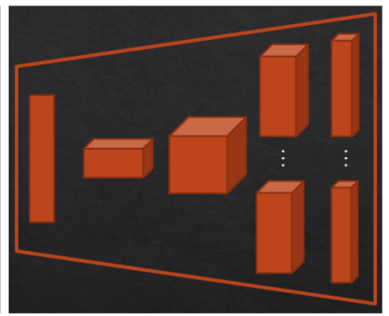

(d) Three layers shared

Fig. 10 Different decoder network architectures for the ablation test

shared layer. This highlights the differences between our proposed GAN framework and the VAE framework presented in [37]. Unlike the VAE network, noise is added when encoding features from an input sketch in our GAN network. As in typical GANs, the noise works as the latent vector and represents the underlying 3D information of the original shape. As a result, the concept of reconstructing the underlying 3D shape by having a common layer for different depth views in the decoder is not needed. Without such an explicit constraint among different views, it can be observed that our proposed framework can still generate high-quality results.

As presented in Sect. 6.3, we evaluated the performance of our proposed method quantitatively. In Fig. 6, the losses of the 4 variants (i.e. sharing $0-3$ layers) of the decoder network are plotted. It can be seen the losses obtained by 0 -layer shared (coloured in red) are the lowest in general. This further justifies our decoder network design.

Finally, we compare our results with those generated by the state-of-the-art method [37] and the results are presented in Fig. 11. The results highlight that our proposed framework generated more realistic results that are closer to the car shape as in the input sketch and contains more fine details on the mesh. In contrast, the meshes generated by [37] are having less distinctive shapes and a lot of artefacts such as holes and noise on the surface.

\section{Conclusion and discussions}

In this paper, we present a system to reconstruct detailed 3D car shapes with a single 2D contour sketch. To effectively learn the correlation between contour sketches and 3D cars, we propose a generative adversarial network (GAN) with an intermediate multi-view depth image representation as to the output and construct the $3 \mathrm{D}$ cars as a post-processing step. To ensure the volume and diversity of the training data, we propose a feature-preserving augmentation pipeline to synthesize more car meshes with realistic sketch-like annotations while keeping the shape of important features. Finally, since deep learning has limited capability in representing fine 

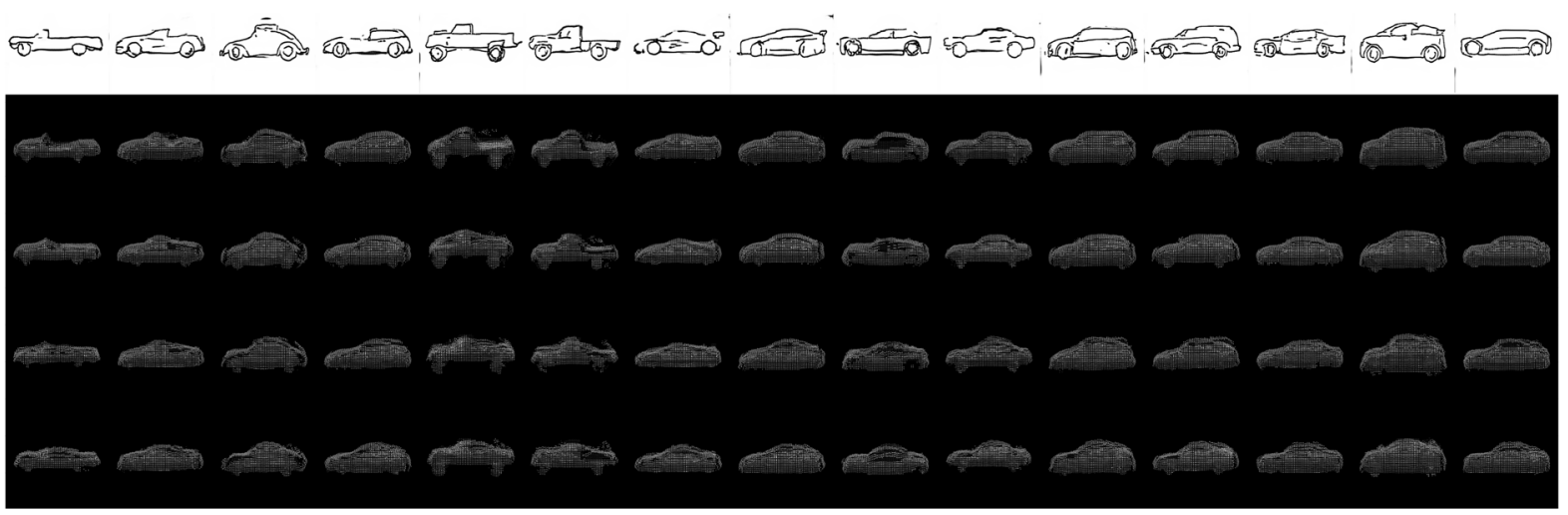

(a) Our proposed GAN network
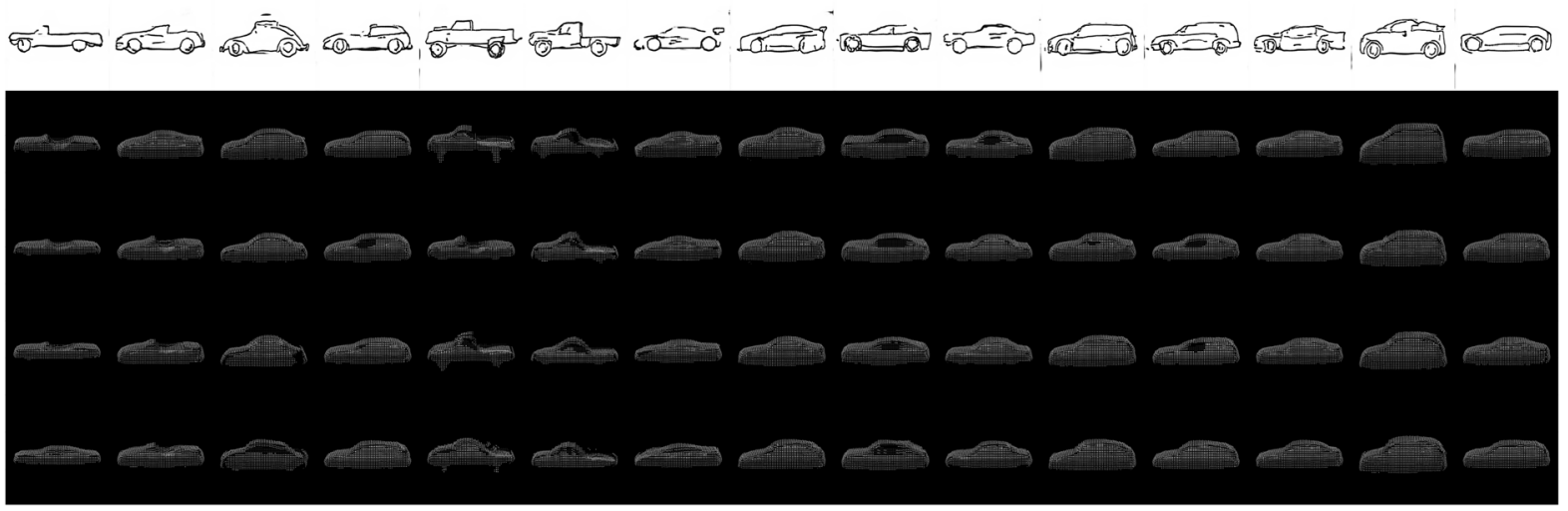

(b) Nozawa et al. (Nozawa et al., 2020)

Fig. 11 The 3D point clouds reconstructed with different decoder architectures. From top to bottom: input sketches, results of the decoder with no shared layer, sharing the first layer, sharing the first two layers, and sharing the first three layers

details, we propose a lazy learning algorithm to construct a small subspace based only on a few relevant database samples for optimizing a car shape with fine detail features. We show that the system performs robustly in creating cars of substantially different shapes and topologies, with realistic detailed features included.

Our main focus in this work is to produce a 3D car models from a single sketch image given by the user. As a result, the proposed framework mainly resembles the exterior shape of the car without considering the configurations of the internal mechanical parts. One of the interesting future direction is to include additional constraints in the 3D car shape generation module to reserve space for the internal car parts.

We use multi-view depth images as an intermediate representation in the generative adversarial network. The two major advantages are that we do not need to deal with $3 \mathrm{D}$ deep learning, which is memory hungry and complicated to train, and we can have a more explicit 2D-to-2D correlation. Currently, we combine the depth images as a post-processing step. However, it is possible to consider them as a means of rectifying the output space and construct extra layers to learn the regression between multi-view depth images and $3 \mathrm{D}$ shapes. One future direction is to explore network archi- tectures for this purpose and introduce more views of depth images in a middle layer of the network for supervision.

The proposed methodology is generic to product design. It is expected that the framework can be applied to producing 3D shapes of other types of products from sketch images. This requires a new data set with paired sketch images and 3D model for the new product type. In the future, we will explore in this direction such as producing 3D furniture models from sketch images with the IKEA data set [32].

Acknowledgements This project was supported in part by the Royal Society (Ref: IES $\backslash$ R2 $\backslash 181024$ and IES $\backslash$ R1 $\backslash 191147$ ), JST ACCEL (JPMJAC1602), JST-Mirai Program (JPMJMI19B2) and JSPS KAKENHI (JP19H01129).

\section{Compliance with ethical standards}

Conflicts of interest The authors declare that they have no conflict of interest

Open Access This article is licensed under a Creative Commons Attribution 4.0 International License, which permits use, sharing, adaptation, distribution and reproduction in any medium or format, as long as you give appropriate credit to the original author(s) and the source, provide a link to the Creative Commons licence, and indi- 
cate if changes were made. The images or other third party material in this article are included in the article's Creative Commons licence, unless indicated otherwise in a credit line to the material. If material is not included in the article's Creative Commons licence and your intended use is not permitted by statutory regulation or exceeds the permitted use, you will need to obtain permission directly from the copyright holder. To view a copy of this licence, visit http://creativecomm ons.org/licenses/by/4.0/.

\section{References}

1. Blanz, V., Vetter, T.: A morphable model for the synthesis of $3 \mathrm{~d}$ faces. In: Proceedings of the 26th annual conference on computer graphics and interactive techniques. SIGGRAPH '99, pp 187-194. ACM Press/Addison-Wesley Publishing Co., New York (1999)

2. Canny, J.: A computational approach to edge detection. IEEE Trans. Pattern Anal. Mach. Intell. 6, 679-698 (1986)

3. Chai, J., Hodgins, J.K.: Performance animation from lowdimensional control signals. ACM Trans. Graph. 24(3), 686-696 (2005)

4. Chang, A.X., Funkhouser, T., Guibas, L., Hanrahan, P., Huang, Q., Li, Z., Savarese, S., Savva, M., Song, S., Su, H., Xiao, J., Yi, L., Yu, F.: ShapeNet: an information-rich 3D model repository. Technical report, Stanford University-Princeton University-Toyota Technological Institute at Chicago (2015) arXiv:1512.03012 [cs.GR]

5. Charles, R.Q., Su, H., Kaichun, M., Guibas, L.J.: Pointnet: deep learning on point sets for 3D classification and segmentation. In: Proceedings of the 2017 IEEE conference on computer vision and pattern recognition (CVPR), pp 77-85 (2017)

6. Chen, Q., Koltun, V.: Photographic image synthesis with cascaded refinement networks. In: Proceedings of the IEEE international conference on computer vision, pp 1511-1520 (2017)

7. Choy, C.B., Xu, D., Gwak, J., Chen, K., Savarese, S.: 3D-r2n2: a unified approach for single and multi-view $3 \mathrm{D}$ object reconstruction. In: The European conference on computer vision (ECCV). Springer, New York (2016)

8. Corsini, M., Cignoni, P., Scopigno, R.: Efficient and flexible sampling with blue noise properties of triangular meshes. IEEE Trans. Vis. Comput. Gr. 18(6), 916-924 (2012)

9. Delanoy, J., Aubry, M., Isola, P., Efros, A., Bousseau, A.: 3D sketching using multi-view deep volumetric prediction. Proc. ACM Comput. Gr. Interact. Tech. 1, 21 (2018)

10. Dinesh Reddy, N., Vo, M., Narasimhan, S. G.: Carfusion: combining point tracking and part detection for dynamic $3 \mathrm{D}$ reconstruction of vehicles. In: Proceedings of the IEEE conference on computer vision and pattern recognition, pp 1906-1915 (2018)

11. Fan, H., Su, H., Guibas, L.J.: A point set generation network for 3D object reconstruction from a single image. In: Proceedings of the IEEE conference on computer vision and pattern recognition, pp 605-613 (2017)

12. Gauthier, M., Poulin, P.: Preserving sharp edges in geometry images. In: Proceedings of graphics interface 2009, GI '09, pp 1-6, Toronto, Canada. Canadian Information Processing Society (2009)

13. Gingold, Y., Igarashi, T., Zorin, D.: Structured annotations for $2 \mathrm{~d}-$ to-3d modeling. CM Trans. Graph. 28(5), 148:1-148:9 (2009)

14. Goedicke, D., Li, J., Evers, V., Ju, W.: Vr-oom: virtual reality onroad driving simulation. In: Proceeding of the $2018 \mathrm{CHI}$ conference on human factors in computing systems, pp 1-11 (2018)

15. Goodfellow, I., Pouget-Abadie, J., Mirza, M., Xu, B., WardeFarley, D., Ozair, S., Courville, A., Bengio, Y.: Generative adversarial nets. In: Advances in neural information processing systems, pp 2672-2680 (2014)
16. Güler, R.A., Neverova, N., Kokkinos, I.: Densepose: dense human pose estimation in the wild. In: Proceedings of the 2018 IEEE/CVF conference on computer vision and pattern recognition, pp 7297$7306(2018)$

17. Han, X., Gao, C., Yu, Y.: Deepsketch2face: a deep learning based sketching system for 3D face and caricature modeling. ACM Trans. Graph. (TOG) 36(4), 126 (2017)

18. Hanocka, R., Metzer, G., Giryes, R., Cohen-Or, D.: Point2mesh: a self-prior for deformable meshes (2020). arXiv:2005.11084

19. Henry, J., Shum, H.P.H., Komura, T.: Interactive formation control in complex environments. IEEE Trans. Vis. Comput. Grap. 20(2), 211-222 (2014)

20. Ho, E.S.L., Shum, H.P.H., Cheung, Y.-M., Yuen, P.C.: Topology aware data-driven inverse kinematics. Comput. Grap. Forum 32(7), 61-70 (2013)

21. Huang, H., Wu, S., Gong, M., Cohen-Or, D., Ascher, U., Zhang, H.: Edge-aware point set resampling. ACM Trans. Graph. (TOG) 32(1), 1-12 (2013)

22. Igarashi, T., Igarashi, T., Hughes, J.F.: Smooth meshes for sketchbased freeform modeling. In: ACM SIGGRAPH: Courses, SIGGRAPH '06, p. 2006. ACM, New York (2006)

23. Igarashi, T., Igarashi, T., Matsuoka, S., Tanaka, H.: Teddy: a sketching interface for 3D freeform design. In: ACM SIGGRAPH: Courses, SIGGRAPH '07, p. 2007. ACM, New York (2007)

24. Isola, P., Zhu, J.-Y., Zhou, T., Efros, A.A.: Image-to-image translation with conditional adversarial networks. In: Proceedings of the IEEE conference on computer vision and pattern recognition, $\mathrm{pp}$ 1125-1134 (2017)

25. Jiang, L., Shi, S., Qi, X., Jia, J.: Gal: geometric adversarial loss for single-view 3D-object reconstruction. In: Proceedings of the European conference on computer vision (ECCV), pp 802-816 (2018)

26. Joshi, P., Carr, N.A.: Repoussé: automatic inflation of $2 \mathrm{~d}$ artwork. In: SBM, pp 49-55. Citeseer (2008)

27. Kingma, D.P., Mohamed, S., Rezende, D.J., Welling, M.: Semisupervised learning with deep generative models. In: Advances in neural information processing systems, pp 3581-3589 (2014)

28. Kraevoy, V., Sheffer, A., Shamir, A., Cohen-Or, D.: Nonhomogeneous, resizing of complex models. In: ACM SIGGRAPH Asia: Papers, SIGGRAPH Asia '08, pp 111:1-111:9, p. 2008. ACM, New York (2008)

29. Li, C., Pan, H., Liu, Y., Tong, X., Sheffer, A., Wang, W.: Robust flow-guided neural prediction for sketch-based freeform surface modeling. ACM Trans. Graph. 37(6), 238:1-238:12 (2018)

30. Li, C., Wand, M.: Precomputed real-time texture synthesis with markovian generative adversarial networks. In: European conference on computer vision, pp 702-716. Springer, New York (2016)

31. Li, M., Lin, Z., Mech, R., Yumer, E., Ramanan, D.: Photosketching: inferring contour drawings from images. In: Proceedings of the 2019 IEEE winter conference on applications of computer vision (WACV), pp 1403-1412. IEEE (2019)

32. Lim, J.J., Pirsiavash, H., Torralba, A.: Parsing IKEA objects: fine pose estimation. ICCV (2013)

33. Lun, Z., Gadelha, M., Kalogerakis, E., Maji, S., Wang, R.: 3D shape reconstruction from sketches via multi-view convolutional networks. In: Proceedings of the 2017 international conference on 3D vision (3DV), pp 67-77. IEEE (2017)

34. Martin, D., Fowlkes, C., Tal, D., Malik, J.: A database of human segmented natural images and its application to evaluating segmentation algorithms and measuring ecological statistics. In: Proceeding of the eighth IEEE international conference on computer vision. ICCV 2001, vol 2, pp 416-423. IEEE (2001)

35. Mirza, M., Osindero, S.: Conditional generative adversarial nets (2014). arXiv preprint arXiv:1411.1784 
36. Nishida, G., Garcia-Dorado, I., Aliaga, D.G., Benes, B., Bousseau, A.: Interactive sketching of urban procedural models. ACM Trans. Graph. (TOG) 35(4), 130 (2016)

37. Nozawa, N., Shum, H.P., Ho, E.S., Morishima, S.: Single sketch image based 3D car shape reconstruction with deep learning and lazy learning. In: VISIGRAPP (1: GRAPP), pp 179-190 (2020)

38. Olsen, L., Samavati, F.F., Sousa, M.C., Jorge, J.A.: Sketch-based modeling: a survey. Comput. Grap. 33(1), 85-103 (2009)

39. Owada, S., Nielsen, F., Nakazawa, K., Igarashi, T., Igarashi, T.A.: Sketching interface for modeling the internal structures of $3 \mathrm{~d}$ shapes. In: ACM SIGGRAPH: courses, SIGGRAPH '06, p. 2006. ACM, New York (2006)

40. Pathak, D., Krahenbuhl, P., Donahue, J., Darrell, T., Efros, A. A.: Context encoders: feature learning by inpainting. In: Proceedings of the IEEE conference on computer vision and pattern recognition, pp 2536-2544 (2016)

41. Qi, C.R., Yi, L., Su, H., Guibas, L.J.: Pointnet++:deep hierarchical feature learning on point sets in a metric space. In: Proceedings of the 31 st international conference on neural information processing systems, NIPS'17, pp 5105-5114, USA. Curran Associates Inc. (2017)

42. Rameau, F., Ha, H., Joo, K., Choi, J., Park, K., Kweon, I.S.: A realtime augmented reality system to see-through cars. IEEE Trans. Vis. Comput. Grap. 22(11), 2395-2404 (2016)

43. Sela, M., Richardson, E., Kimmel, R.: Unrestricted facial geometry reconstruction using image-to-image translation. In: Proceedings of the 2017 IEEE international conference on computer vision (ICCV), pp 1585-1594 (2017)

44. Shao, C., Bousseau, A., Sheffer, A., Singh, K.: Crossshade: shading concept sketches using cross-section curves. ACM Trans. Graph. (SIGGRAPH Conf. Proc.) 31, 4 (2012)

45. Shen, Y., Henry, J., Wang, H., Ho, E.S.L., Komura, T., Shum, H.P.H.: Data-driven crowd motion control with multi-touch gestures. Comput. Grap. Forum 37(6), 382-394 (2018)

46. Shen, Y., Yang, L., Ho, E.S.L., Shum, H.P.H.: Interaction-based human activity comparison. IEEE Trans. Vis. Comput. Grap. (2019)

47. Shtof, A., Agathos, A., Gingold, Y., Shamir, A., Cohen-Or, D.: Geosemantic snapping for sketch-based modeling. Comput. Graph. Forum 32(2pt2), 245-253 (2013)

48. Shum, H.P.H., Ho, E.S.L., Jiang, Y., Takagi, S.: Real-time posture reconstruction for microsoft kinect. IEEE Trans. Cybern. 43(5), 1357-1369 (2013)

49. Tatarchenko, M., Dosovitskiy, A., Brox, T.: Octree generating networks: efficient convolutional architectures for high-resolution $3 \mathrm{~d}$ outputs (2017)

50. Umetani, N.: Exploring, generative 3D shapes using autoencoder networks. In: SIGGRAPH Asia: Technical Briefs, SA '17, pp 24:124:4, p. 2017. ACM, New York (2017)

51. Wang, T., Liu, M., Zhu, J., Tao, A., Kautz, J., Catanzaro, B.: High-resolution image synthesis and semantic manipulation with conditional gans. In: Proceedings of the 2018 IEEE/CVF conference on computer vision and pattern recognition, pp 8798-8807 (2018)

Publisher's Note Springer Nature remains neutral with regard to jurisdictional claims in published maps and institutional affiliations.

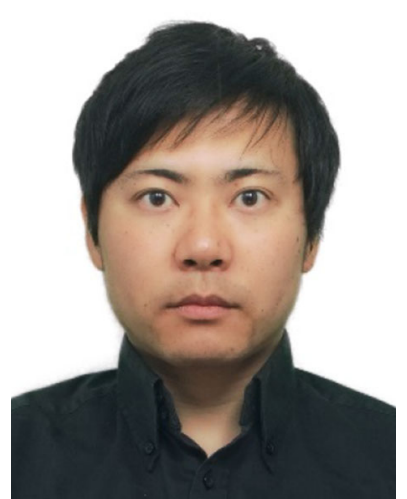

Naoki Nozawa is currently pursuing his $\mathrm{PhD}$ degree in Waseda University. $\mathrm{He}$ received the $\mathrm{BE}$ and ME in Applied Physics from the Graduate School of Advanced Science and Engineering at Waseda University, Tokyo, Japan, in 2015 and 2017, respectively. His main research area includes deep learning applications, computer vision and computer graphics.

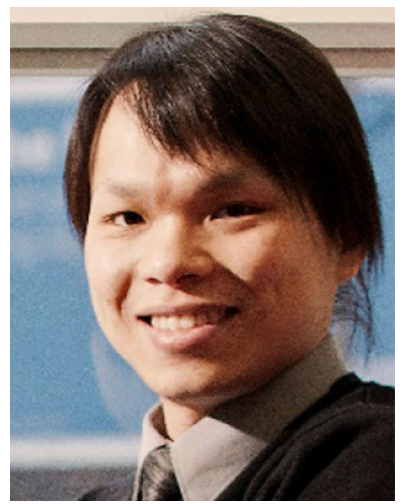

Hubert P. H. Shum is an Associate Professor in Computer Science at Durham University. Before this, he worked as the Director of Research, Associate Professor, Senior Lecturer at Northumbria University, and a Postdoctoral Researcher at RIKEN Japan. He received his $\mathrm{PhD}$ degree from the University of Edinburgh. He published over 100 research papers in the fields of computer graphics, computer vision, motion analysis and machine learning.

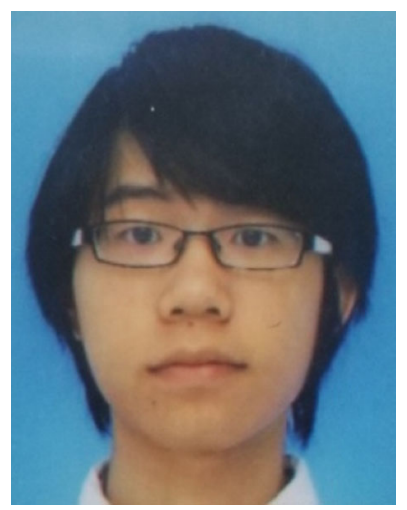

Qi Feng is currently pursuing his $\mathrm{PhD}$ degree in Waseda University. $\mathrm{He}$ received the $\mathrm{BE}$ and $\mathrm{ME}$ in Applied Physics from the Graduate School of Advanced Science and Engineering at Waseda University, Tokyo, Japan, in 2017 and 2019, respectively. His main research area includes deep learning applications, computer vision, computer graphics, virtual and augmented reality.

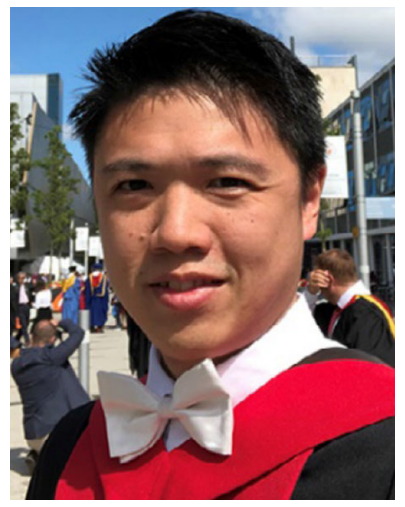

Edmond S. L. Ho is currently a Senior Lecturer in the Department of Computer and Information Sciences at Northumbria University, Newcastle, UK. Prior to joining Northumbria University in 2016, he was a Research Assistant Professor in the Department of Computer Science at Hong Kong Baptist University. He received the $\mathrm{PhD}$ degree from the University of Edinburgh. His research interests include Computer Graphics, Computer Vision, and Machine Learning. 


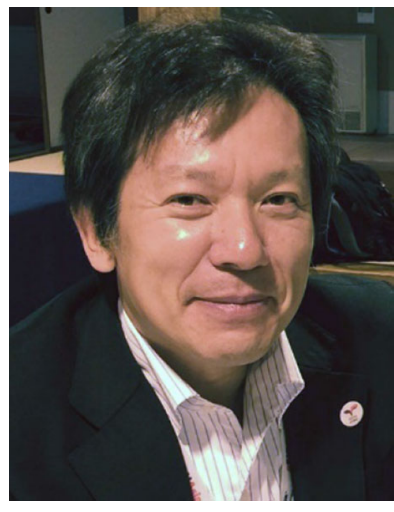

Japan Award in 2020.
Shigeo Morishima is a Professor of Graduate School of Advanced Science and Engineering, Waseda University in Japan. He received the $\mathrm{BS}, \mathrm{MS}$, and $\mathrm{PhD}$ degrees, all in Electrical Engineering from the University of Tokyo, Japan, in 1982, 1984, and 1987, respectively. From 1987 to 2001, he was an Associate Professor and from 2001 to 2004 a Professor of Seikei University. His research interest includes Computer Graphics, Computer Vision and Human Computer Interaction. He won the CG 